\title{
A New CM T Flex Aphakia Foldable IOL with Trabeculectomy for a Case of Traumatic Aphakia with Secondary Glaucoma
}

\author{
Nivean $\mathbf{M}^{1}$, Murali Ariga ${ }^{2}$, Pratheeba Devi Nivean ${ }^{3}$ and Archana $\mathbf{N}^{4 *}$ \\ ${ }^{1}$ Vitreoretinal Department, M.N. Eye Hospitals, India \\ ${ }^{2}$ Glaucoma Department, M.N. Eye Hospitals, India \\ ${ }^{3}$ Oculoplasty Department, M.N. Eye Hospitals, India \\ ${ }^{4}$ Cataract and Medical Retina, M.N. Eye Hospitals, India \\ *Corresponding Author: Archana N, Cataract and Medical Retina, M.N. Eye \\ Hospitals, India.
}

\begin{abstract}
Here, we report a case of 37 year old male who came with a history of traumatic aphakia in the right eye 10 years back along with uncontrolled intraocular pressure (IOP). His Uncorrected vision was hand movements in the right eye at presentation. He was managed with new CM T-FLEX foldable aphakic scleral fixated intraocular lens with superior trabeculectomy. Fornix based conjunctival partial thickness triangular scleral flap of $4 \mathrm{~mm}$ were raised. Mitomycin c was placed for 1 minute. Surgical iridectomy was done using Kellys punch. Flap closed with releasable sutures. Two partial-thickness limbal-based scleral flaps of $2.5 \mathrm{~mm} * 2.5 \mathrm{~mm}$ were created 180 degree apart; sclerotomies were made using $23 \mathrm{G}$ needle $1.5 \mathrm{~mm}$ away from limbus. The new CM T-flex intraocular lens was injected through the $2.8 \mathrm{~mm}$ clear corneal incision and the specially designed $\mathrm{T}$ shaped lens haptics were externalised with the 23G PraNiv T flex forceps, brought out and left under the scleral flap and using the hand shake technique the second arm was grasped using the NiShi Grasping forceps and exchanged to the PraNiv T flex forceps and brought out through the other sclerostomy. The intraocular lens was stable. Anterior chamber wash was done and air bubble was injected. Fibrin glue was used to close the scleral flaps and conjunctiva. Postoperative period was uneventful. At the end of 1 month, patient's best corrected visual acuity was 6/24 in the right eye with a good filtering bleb superiorly and a stable, well centered CM T flex Aphakia intraocular lens.

Keywords: Secondary Intro Ocular Lens; CM-T Flex Foldable Lens; Secondary Glaucoma; Traumatic Aphakia; Vitrectomy; Trabeculectomy
\end{abstract}

\section{Introduction}

Secondary intraocular lens (IOL) implantation has evolved over the past few decades. Several new techniques, lens options, and materials now exist. Careful patient selection is important to determine the optimal secondary IOL technique. Intraocular lens placement in the capsular bag is the most ideal followed by sulcus placement. However, the best option when no capsular support exists in an aphakic patient remains unclear. Surgeons should be aware of contraindications for each technique; however, there are several situations where anterior chamber intraocular lens (ACIOL) [1], scleral-fixated intraocular lens (SFIOL), and iris fixation can all be used. Among lenses implanted in the anterior chamber, the options include iris-suture-fixated lenses [2] and angle-supported anterior chamber phakic intraocular lenses [3], which are rarely used due to complications, such as endothelial loss, pupil distortion and secondary glaucoma [4]. In those cases, surgeon familiarity and comfort with the secondary IOL technique can determine the type of surgery performed. Recently, sutureless SFIOLs have become popular and can be fixated using different techniques such as the Scharioth technique, Narang and Agarwal's glued SFIOL [5,6]. Yamane's., et al. double-flanged technique [7], or Baskaran's., et al. X-Nit technique [8]. Many modifications of these techniques have also been described using needles, trocars, flaps, sutures, and glue. Iris fixated IOLs including haptic suturing to the iris stroma and retropupillary iris-claw IOL placement has also been described with results similar to sutureless SFIOLs. However, the scleral fixation of an IOL can also lead to many complications such as a decrease in the corneal endothelial cell density $[9,10]$, refractive er- 